\title{
Alfabetización inicial bilingïe e inclusión en la escuela primaria regular
}

\author{
Initial bilingual literacy and inclusion in regular elementary school
}

\author{
Efrén Viramontes Anaya - Ameyalli Porras Palacio
}

Efrén Viramontes Anaya. Escuela Normal Rural Ricardo Flores Magón, Chihuahua, México. Cuenta con estudios como maestro en Educación Campo Práctica Docente por la Universidad Pedagógica Nacional y como doctor en Ciencias de la Educación por el Instituto Pedagógico de Estudios de Posgrado. Tiene los reconocimientos al perfil Prodep desde el año 2011 y del Sistema Nacional de Investigadores a partir del 2018. Presidente de la Red Nacional de Formación Docente 2015-2019. Su producción académica principal es sobre formación docente, evaluación del aprendizaje y enseñanzaaprendizaje de la lengua. Actualmente es profesor-investigador de tiempo completo en la Escuela Normal Rural Ricardo Flores Magón y catedrático de posgrado en la Universidad Pedagógica Nacional del estado de Chihuahua. Correo electrónico: efren8000@hotmail.com. ORCID: https:// orcid.org/ 0000-0003-4378-3095.

Ameyalli Porras Palacio. Servicios Educativos del Estado de Chihuahua, México. Es docente en la Escuela Primaria Federal Xicoténcatl de Ciudad Juárez, Chihuahua. Es egresada de la Escuela Normal Rural Ricardo Flores Magón como licenciada en Educación Primaria, con formación bilingüe. Actualmente su línea de estudio
Resumen

Esta investigación aborda la temática de la alfabetización inicial bilingüe en una escuela primaria regular. Se hace un análisis del proceso de una niña de la etnia tarahumara, en un grupo de primer grado de la educación primaria regular. El propósito investigativo es el de analizar el aprendizaje de la lectura y escritura mediante una metodología de estudio de caso intrínseco, que es complementado con aportaciones de opiniones de docentes a través de entrevistas. Los resultados se centran en las acciones didácticas y los avances académicos de la alumna, sobre el desarrollo de la lectura y la escritura en lengua materna rarámuri o rarámuli, así como en el español como segunda lengua. Se precisan los avances que se tienen en el aula y en el hogar de la estudiante, señalando los aciertos y desaciertos de la metodología de enseñanza empleada. Se concluye con una propuesta concreta basada en la realización de un proceso didáctico que considere el contexto y el lenguaje de la estudiante y su competencia lingüística para el desarrollo de la alfabetización bilingüe, con un enfoque comunicativo.

Palabras clave: Alfabetización, interculturalidad, bilingüismo, inclusión educativa, escritura.

\section{Abstract}

This research addresses issues regarding the first stages of bilingual literacy in a primary school. An analysis of the process is made in a girl belonging to the Tarahumara ethnic group; in a group of the first grade of regular primary education. The purpose is to analyze the learning of reading and writing through an intrinsic case study methodology, which is complemented with contributions from teachers' opinions based on interviews. The results focus on the didactic actions and academic progress of the student, concerning the development of reading and writing in the mother tongue Rarámuri or Rarámuli, as well as in Spanish as a second language. The progress made in the classroom and the student's home is shown, pointing out the successes and failures of the methodology used, con- 
es la educación bilingüe en educación primaria. Correo electrónico: ame160210@gmail.com. ORCID: https://orcid.org/ 0000-0001-76944911. cluding with a concrete proposal based on the realization of a didactic process that considers the context and the language of the student and the linguistic competence she has for the development of bilingual literacy, with a communicative approach.

Keywords: Literacy, interculturality, bilingualism, educational inclusion, writing.

\section{INTRODUCCIÓN}

Esta investigación trata temáticas referidas a la educación intercultural y a la alfabetización inicial en lengua materna y segunda lengua en una escuela regular de educación primaria. Se desarrolló en un grupo de primer grado grupo, con énfasis al estudio de un caso en particular, en la Escuela Primaria Lázaro Cárdenas ubicada en la localidad de Lázaro Cárdenas, de Meoqui, Chihuahua.

Durante el desarrollo de la investigación se nombra al caso estudiado de dos formas, Caso X o alumna, para conservar el anonimato; de igual manera se hará mención del español como segunda lengua, además de lengua materna al rarámuri, y los niños que pertenecen a una etnia indígena serán mencionados como tales o niños indígenas.

Existen fundamentos legales en México que ofrecen las condiciones para que los ciudadanos de las etnias y grupos vulnerados accedan y permanezcan en el sistema educativo nacional, como lo establecen el artículo tercero constitucional y la Ley General de Educación, los artículos $2^{\circ}$ y 38 también apoyan esta idea.

En esta investigación se plantea como problema principal el proceso de alfabetización bilingüe del Caso X, quien cursa primer grado de primaria y su lengua materna es el rarámuri o ralámuri (perteneciente a la etnia tarahumara de Chihuahua, México), con quien se pretende que aprenda a leer y a escribir en español, que es su segunda lengua, cuyo desarrollo es apenas incipiente. El hecho se desarrolla en una escuela primaria regular de la región centro sur del estado de Chihuahua.

Se decidió realizar la presente investigación en torno a conocer: ¿Cómo se desarrolla el proceso de construcción de la alfabetización bilingüe de un caso de etnia tarahumara? Con este planteamiento, el objetivo es indagar sobre los elementos que se encuentran a alrededor de la alfabetización bilingüe inicial de un caso específico, en un ambiente intercultural de una escuela primaria regular: analizando el proceso, identificando las barreras para el aprendizaje y la atención por parte de los docentes, así como la descripción del proceso didáctico vivido en el aula, el albergue y el contexto familiar.

Como parte de la revisión de la literatura se destacan las aportaciones de Martínez (2015) sobre los marcos normativos en México de la educación indígena; de Schmelkes (2013), con el tema de la interculturalidad en la escuela, como un espacio de confluencia de varias culturas que interactúan, así como el análisis de la migración de pueblos indígenas a espacios culturales más urbanizados. De Carrillo (2012), con- 
tribuyó respecto a la situación de la formación docente para el medio indígena, en relación con la enseñanza intercultural bilingüe y las deficiencias más importantes.

La alfabetización inicial es uno de los elementos que son obligados conceptualizar. Manghi, Crespo, Bustos y Haas (2016) la definen como

...proceso de cambio en el propio individuo que se alfabetiza -el aprendizaje-, es decir, aquel que se evidencia en la adquisición de ciertos saberes (conceptos, habilidades o valores) que se producen en el sujeto y que le permiten participar en actividades letradas de su comunidad. En una interpretación más semiótica, la transformación tiene que ver con un proceso de aprender a entender el mundo desde determinadas convenciones culturales, como las que conlleva la lengua escrita [p. 81].

La alfabetización es parte inherente de las actividades culturales del contexto, a través de las prácticas sociales del lenguaje, considerando este proceso como una acción en el ámbito social y en el cognitivo, que el niño realiza en el uso funcional, reflexivo y práctico de la lectura, la escritura, el habla y la escucha.

Esta idea le da sentido a la alfabetización bilingüe que está implicada en la investigación, sobre todo porque que el caso que se estudia su lengua materna indígena, y que su segunda lengua, el español, la ha desarrollado incipientemente. Por lo tanto, al considerase como proceso cognitivo y social del que hacen los especialistas de la literatura antes citada, se tiene que establecer un dispositivo didáctico que atienda estas características.

La escritura se considera como una forma de representación del pensamiento y la oralidad por medio de grafías estructuradas en un sistema socialmente establecido (Piacente, 2010; Querejeta, Piacente, Guerrero y Alva, 2013), este sistema, como lo explica Vygotsky (1979), se compone de "signos de relaciones y entidades reales" (p. 160), en uso contextualizado, con fines comunicativos, para que todo acto de escritura sea un acto de comunicación.

Solé (2019) explica la lectura como el proceso interactivo entre el texto y el lector, para construir el significado de las ideas que expone el autor, relacionándolo con lo que conoce sobre el tema, reconstruyendo el texto, poniéndose en condiciones de opinar sobre su contenido y de usar la información leída en su propio contexto.

Jiménez (2014) agrega que leer es "el proceso mediante el cual se comprende el texto escrito" (p. 3). De acuerdo con estos especialistas en la lectura, esta actividad implica mucho más que la simple decodificación, es más una construcción que depende del marco referencial del lector y de lo que le ofrece el texto como reto para obtener significado mediante dicha interacción. Por ello la comprensión y la lectura supera el contenido literal de un texto, así como la simple decodificación de signos.

En cuanto a la interacción intercultural, Hidalgo (2005) señala que implica "una comunicación comprensiva entre las distintas culturas que conviven en un mismo espacio, siendo a través de estas donde se produce el enriquecimiento mutuo [...] el reconocimiento y la valoración [...] de cada una de las culturas en un marco de 
igualdad" (p. 78); en este proceso el lenguaje tiene un papel muy importante, a través de las interacciones lingüísticas y culturales se busca que el estudiantado logre un aprendizaje de su segunda lengua y simultáneamente se promueva el enriquecimiento de la lengua materna y transmisión de conocimiento entre todo el grupo.

Viramontes, Morales y Burrola (2011) presentan un análisis del desarrollo de la escritura del español como lengua no materna del educando, con niños indígenas tarahumaras bilingües, y de las dificultades que enfrentan tanto el docente como el alumno en el proceso de alfabetización en una escuela primaria indígena y otra regular, aportando la idea de que la cultura de los niños, como en este caso de la niña de etnia tarahumara, la interacción entre las culturas o procesos interculturales provocados por la escuela representan una forma de atención a la diversidad y a la valoración de los saberes culturales de los alumnos, como es el caso de la lengua indígena materna.

La interculturalidad en el aula es una gran oportunidad de aprendizaje para las culturas que existen en el grupo escolar, para Schmelkes este proceso micro social y pedagógico es "una aspiración, no una realidad, preferimos hablar de educación para la interculturalidad en lugar de educación intercultural. La educación debe contribuir - y puede hacerlo de manera privilegiada- a la construcción de una nación intercultural" (Schmelkes, 2013, p. 6), en este caso la aspiración es todavía menor, se circunscribe de manera concreta a lo que sucede en el aula, en el grupo escolar que cada docente tiene, se refiere a usar el salón de clase como un espacio común en el que las culturas pueden coexistir y manifestarse, creando relaciones de aprendizaje conjuntas, derivadas de la interacción cultural como un contenido transversal en el currículo escolar.

Estas posturas teóricas, aplicadas a las interacciones en el aula, abren un panorama esperanzador para los niños que han vivido la segregación y discriminación en el ámbito social y escolar por causa de diferencia de raza, estatus social, religión etc., y ofrecen un elemento que enriquece un proyecto alternativo pedagógico incluyente.

En lo que corresponde a la educación intercultural bilingüe, Schmelkes (2013) explica que este tipo de educación tiene la finalidad de que "los indígenas conozcan y valoren su cultura y dominen su lengua, pero que a la vez descubran la riqueza de los otros grupos culturales que habitan el planeta" (p. 28); junto con esto plantea la idea de que su aplicación no es exclusiva hacia los grupos sociales minoritarios, sino a la población en general, para que la interculturalidad se dé en toda la sociedad, no de un grupo a otro, más bien entre grupos culturales diferentes que conviven en un territorio. Esto es justamente lo que posibilita que los niños de un grupo escolar aprovechen mutuamente las aportaciones y conocimientos culturales, como la lengua, para interactuar de manera más humana y respetuosa.

En otras aportaciones que complementan teóricamente estos procesos alfabetizadores, Morin (1995) explica la posibilidad de lograr la interacción intercultural a través de los principios del pensamiento complejo, atendiendo la problemática de la 
inclusión, la interculturalidad y la alfabetización como elementos que se encuentran intrincados en un mismo sistema de hechos interconectados. Esto implica comprender holísticamente la alfabetización bilingüe, así como las acciones que se realicen en torno a su desarrollo, considerando a la educación en el aula escolar como una oportunidad de atención a las necesidades generales de los educandos, en contexto incluyente y formativo.

\section{Planteamiento metodológico}

El paradigma interpretativo es el que se consideró más apropiado para implementar en esta investigación, ya que se busca describir, analizar e interpretar los resultados para comprender el proceso de la alfabetización inicial. El enfoque es cualitativo; el método es de estudio de casos, como lo plantea Stake (2005), tipificado como caso intrínseco, complementado con la teoría fundamentada de Strauss y Corbin (2016) y Bonilla y López (2016). En cuanto a las técnicas de investigación, se aplicaron la observación participante (Monje, 2011) en el contexto de trabajo en aula de la estudiante; una encuesta (Arias y Covinos, 2021) que se aplicó a 40 docentes de la zona de trabajo, con la intención de conocer a quiénes habían impartido clases a niños de grupos étnicos para ser entrevistados posteriormente; la entrevista semiestructurada (Rodríguez, 2011) aplicada a cinco docentes seleccionados, considerando los datos de la encuesta, con el propósito de conocer más sobre el proceso de construcción de la alfabetización en segunda lengua de niños de grupos étnicos. Los instrumentos utilizados fueron el cuestionario de preguntas cerradas, que sirvió para dar a conocer de una manera rápida los participantes seleccionados para la entrevista y ampliar el tiempo en la misma, y el diario de campo, que contribuyó para conocer el desarrollo de la lectura y escritura, logros y hallazgos de contraste con ambas lenguas que fueron surgiendo (Hernández-Sampieri y Mendoza, 2018).

El procesamiento o análisis de la información se realizó de acuerdo con lo establecido por Strauss y Corbin (2016) y Bonilla y López (2016) mediante las tres fases propuestas similares a la descripción, ordenamiento conceptual y teorización. Primero se realizó una descripción de la evolución que tuvo el caso, un ordenamiento conceptual de la información clasificándola en distintas categorías que engloban tanto los entornos como los factores que se considera que influyen para el desarrollo adecuado de la alfabetización inicial del caso. Por último se realizó un contraste del análisis realizado y las opiniones teóricas al respecto, estructurando las categorías que responden al propósito de la investigación.

\section{Resultados DE LA INVESTIGACIÓN}

El primer asunto que se analiza del Caso X es lo relacionado con la inclusión y la discriminación en la escuela. Sobre esto se tuvo la información de que durante la 
educación preescolar hubo eventos discriminatorios y de exclusión que le marcaron la vida, esto se hizo evidente por la observación de la poca interacción que la alumna tenía en la clase, que afectaba su proceso de adaptación, socialización y descubrimiento del lenguaje. Realizaba las actividades orales con una voz que apenas se percibía para su propio oído, no le gustaba que la escucharan hablar y no se sentía en tanta confianza con ellos, esto evitaba que fuera desarrollando el proceso de lectura y escritura de manera semejante ya a la misma velocidad que el resto del grupo.

Se cree que este comportamiento se derivaba, en parte, por la interacción con sus compañeros, que realizaban prácticas discriminatorias observadas al interior del albergue de algunas compañeras de otros grupos y una compañera del mismo salón hacia ella.

Para los últimos días que se trabajó con el grupo ya había más interacción con sus compañeros, era ella quien comenzaba conversación con los alumnos, y ya no solo con aquellos que se sentaban a su lado.

A partir del tratamiento del asunto de discriminación e inclusión del Caso X, en el grupo se tuvieron eventos en los que sus compañeros la valoraron más como compañera, evitando excluirla y discriminarla; el trato fue cambiando gradualmente, sobre todo porque la maestra les demostró que en lugar de ser una desventaja hablar una lengua materna indígena y, aunque más incipiente, el español, significaba una virtud, como sucedía con una alumna que también hablaba el inglés como lengua materna, ambas podían comunicarse en dos idiomas, mientras que el resto del grupo solo en uno.

Se consideró pertinente abordar la interculturalidad no solo para favorecer la alfabetización sino para realizar actividades encaminadas a la valoración y respeto a la diversidad, para evitar que este tipo de prácticas se repitieran. Algo que también facilitó este proceso fue el hecho de permitirle el uso del vestuario de su cultura originaria, respetando con ello la diversidad cultural.

Otra categoría de análisis es lo relacionado con la metodología de enseñanza. En un primer momento se consideró el método de enseñanza como un factor que intervenía en el proceso de desarrollo de alfabetización del caso de estudio, el bilingüismo que presenta, por lo que se contrastaron las metodologías de enseñanza que son comúnmente implementadas por los docentes; se llegó a la conclusión de que la metodología aplicada al grupo incluía experiencias previas que la alumna había tenido con el lenguaje y la mayor parte de conocimientos previos que tenía en su lengua materna.

Se identificó que los métodos o enfoques de enseñanza de la alfabetización inicial que son de marcha sintética fueron menos adecuados para comenzar la alfabetización que los globalizadores, en estos últimos se piensa que los enfoques contextualizados que parten del lenguaje de los niños, así como los significados y usos que le dan, son los más efectivos y adecuados para el aprendizaje. En este caso fue la consideración de que existe un marco de referencia y experiencia de lenguaje en rarámuri y otro 
tipo de conocimiento y uso del lenguaje en español. El primero se trató en el ámbito familiar, donde le apoyaron en la alfabetización en su lengua materna, y en lo que corresponde a la segunda lengua, esta fue tratada en la escuela junto con los demás niños del grupo.

En lo que corresponde a la lectura y escritura en lengua materna, la madre y la hermana del Caso X estaban enseñando las bases de la alfabetización en rarámuri, en este caso el lenguaje hablado fue el punto de partida del proceso de alfabetización. También ayudó que en la escuela se le pedía que leyera y escribiera en ambas lenguas.

Se aplicaron en el proceso los dos principios alfabéticos que Flores (2020) sugiere que pueden ser implementados para abordar la alfabetización inicial, desde un enfoque basado en la psicolingüística y en el constructivismo. También fue ampliamente considerado el enfoque intercultural bilingüe, que ayuda a desarrollar destrezas orales en segunda lengua. Siempre se partió del análisis de unidades complejas, en específico de la palabra y de enunciados.

El segundo principio consistió en establecer relación entre escritura e imagen de las palabras que no sean parte de su contexto, en ambos idiomas; posteriormente se traslapa al español ya contextualizado. Este fue implementado durante el último mes que se trabajó con el Caso X, lo cual contribuyó a que adquiriera un mayor nivel de compresión de lo que lee. Además, a través de este principio se fomentó la práctica de la lengua materna de forma oral. Sobre esto es más recomendable atender a los principios constructivistas, como lo propone Vernon (2004) en relación con el proceso de aprovechar siempre lo que el niño ya sabe, para iniciar con el planteamiento de tareas que provoquen el desarrollo de la conciencia de la lógica del sistema de escritura, además de la contextualización del proceso en el uso cultural del lenguaje y proyectarlo hacia el uso convencional y comunicativo en la cultura en la que está accediendo.

En cuanto a la evolución académica del Caso X, al inicio del ciclo escolar se encontraba en la etapa presilábica de construcción de la escritura, fua hasta el tercer bimestre del ciclo escolar, en el que avanzó a la etapa silábico-alfabética, se cree que fue gracias a las actividades encaminadas a favorecer el respeto y reconocimiento de la diversidad, que la alumna se sintiera incluida y no discriminada, lo que generó también una participación activa en clase y con sus compañeros. Para este logro, las actividades complementarias que se realizaron en casa, con ayuda de su hermana y su madre, fueron cruciales.

\section{Conclusiones}

A lo largo del desarrollo de esta investigación se caracterizó el proceso de construcción de la alfabetización inicial en segunda lengua de una infante indígena y, a pesar de que los factores que pueden influir en el desarrollo de alfabetización de un solo caso puede que no sean los mismos que afectan a un sector de la población, en general 
algunos de los hallazgos y resultados que aquí se presentaron pueden ser tomados en cuenta en otros casos.

Se logró conocer de manera general cuáles eran las barreras y dificultades de aprendizaje que se presentaban más frecuentemente en niños indígenas, gracias a las aportaciones de los docentes que colaboraron con la investigación.

Se encontró que existe coincidencia en que el desarrollo de la alfabetización en niños indígenas es más lento que en el resto del grupo, ahora se comprende que esto es consecuencia de la dificultad que representa el bilingüismo, la bi-alfabetización, en su lengua materna y en español, de los efectos de las experiencias previas de discriminación, además de la falta de apoyo extraescolar que generalmente reciben en el albergue.

Se comprendió cómo se desarrolla el aprendizaje de una segunda lengua, considerando como principio que todo conocimiento previo servirá como base para construcción de conocimientos futuros. Es necesario partir de las competencias comunicativas que los alumnos tienen en su lengua materna para desarrollar la alfabetización en segunda lengua y sentar aprendizajes que sean significativos y cobren relevancia, aplicando de esta forma una secuencia en la implementación de los métodos, primero los globalizadores para crear la relación palabra-imagen, que contribuye a la contextualización en su lengua materna, posteriormente los de marcha sintética para establecer la relación grafofonética de la segunda lengua, atendiendo de esta forma la diversidad lingüística, aunque es preferible alfabetizar en la segunda lengua en el mismo proceso de aprendizaje y uso del de la misma.

Hace falta crear más concientización en las escuelas sobre la diversidad cultural y lingüística que se presenta en los centros escolares y el respeto a la misma, para evitar cualquier acto de discriminación que afecte tanto la autoestima de los alumnos como su desempeño escolar. También se requiere aprovechar la gran riqueza que puede brindar la diversidad, para promover el respeto y la empatía en los alumnos, evitando con ello prácticas de exclusión dentro de las aulas.

Se resalta la importancia de tener una comunicación asertiva como docente, con el alumno y su familia, para incentivar el apoyo y motivación hacia sus hijos a realizar su mayor esfuerzo en seguir adquiriendo los aprendizajes necesarios para acceder a niveles de escolarización más altos y promover la preservación de su lengua materna, tanto de manera oral como escrita, mediante situaciones comunicativas reales y útiles.

Estos factores, que la mayoría de los docentes pueden ver como barreras de aprendizaje, fueron los que se rescataron y analizaron para buscar las formas en que se podían utilizar para favorecer la construcción de la alfabetización en segunda lengua y fomentar el desarrollo alfabético de su lengua materna. 


\section{REFERENCIAS}

Arias, J. L., y Covinos, M. (2021). Diseño y metodología de la investigación. Arequipa, Perú: Enfoques Consulting EIRL. Recuperado de: https://www.researchgate.net/publication/352157132_ DISENO_Y_METODOLOGIA_DE_LA_INVESTIGACION.

Bonilla G., M. Á., y López S., A. D. (2016). Ejemplificación del proceso metodológico de la teoría fundamentada. Cinta Moebio, (57), 305-315. Recuperado de: https://www.scielo.cl/ pdf/cmoebio/n57/art06.pdf.

Carrillo, J. (2012). Necesidades de formación docente para la educación intercultural bilingüe en el contexto indigena del estado de Durango, México [Tesis de Doctorado]. Universidad Complutense de Madrid, España. Recuperado de: http://eprints.ucm.es/16674/1/T34003.pdf.

Flores, M. L. (2020). Prácticas evolutivamente adecuadas para aprender a leer y escribir. Recuperado de: https://www.educacioninicial.com/c/002/086-principio-alfabetico/.

Hernández-Sampieri, R., y Mendoza, C. P. (2018). Metodología de la investigación. México: McGraw-Hill Educación.

Hidalgo, H. V. (2005). Cultura, multiculturalidad, interculturalidad y transculturalidad evolución de un término. Revista de Ciències de L'educació, (1), 75-85. Recuperado de: http:// aularedim.net/wp-content/uploads/cultura_multiculturalidad.pdf.

Jiménez P., E. (2014). Comprensión lectora vs competencia lectora: qué son y qué relación existe entre ellas. Revista ISL, (1), 65-74. Recuperado de: https://www.redalyc.org/ pdf/4462/446243919005.pdf.

Manghi, D., Crespo, N., Bustos, A., y Haas, V. (2016). Concepto de alfabetización: ejes de tensión y formación de profesores. Revista Electrónica de Investigación Educativa, 18(2), 79-92. Recuperado de http://redie.uabc.mx/redie/article/view/1038.

Martínez, B. E. (2015). La educación intercultural y bilingüe (EIB) en México. ¿El camino hacia la construcción de una ciudadanía democrática? Relaciones. Estudios de Historia y Sociedad, 36(14), 103-131. Recuperado de: http://www.scielo.org.mx/scielo.php?script=sci_artte xt\&pid=S0185-39292015000100103.

Martínez, R. A. (2011). Métodos de investigación cualitativa. Revista de la Corporación Internacional paraelDesarrollo Educativo, (8), 1-33. Recuperado de: https:/ / www.academia.edu/22207038/ Revista_de_la_Corporaci\%C3\%B3n_Internacional_para_el_Desarrollo_Educativo_ Bogot $\%$ C3\%A1_Colombia_M\%C3\%89TODOS_DE_INVESTIGACI\%C3\%93N_ CUALITATIVA_QUALITATIVE_RESEARCH_METHODS.

Monje A., C. A. (2011). Metodología de la investigación cuantitativa y cualitativa: guia didáctica. Neiva, Colombia: Universidad Surcolombiana. Recuperado de: https://www.uv.mx/rmipe/ files/2017/02/Guia-didactica-metodologia-de-la-investigacion.pdf.

Morin, E. (1995). Introducción al pensamiento complejo. Barcelona: Gedisa.

Piacente, I. T. (2010). Fundamentos, técnicas e instrumentos de exploración psicológica I. UNLPFaHCE-Facultad de Psicología. Recuperado de: https://www.memoria.fahce.unlp.edu. ar/programas/pp.6522/pp.6522.pdf.

Querejeta, M., Piacente, T., Guerrero, B., y Alva, E. A. (2013). La separación entre palabras en la escritura de niños que inician la escolaridad primaria. Interdisciplinaria, 30(1), 45-64. Recuperado de: https://www.redalyc.org/articulo.oa?id=18027808003.

Rodríguez G., D., y Valldeoriola R., J. (2011). Metodología de la investigación. Barcelona: Eureca Media, SL. 
Schmelkes, S. (2013). Educación para un México intercultural. Sinéctica, (40). Recuperado de: http://www.scielo.org.mx/scielo.php?script=sci_arttext\&pid=S1665109X2013000100002.

Solé, I. (2019). Leer, comprender y aprender. Propuestas para favorecer el aprendizaje a partir de textos. España: Cuadernos de Educación.

Stake, R. F. (2005). Investigación con estudio de casos. España: Morata.

Strauss, A., y Corbin, J. (2016). Bases de la investigación cualitativa. Técnicas y procedimientos para desarrollar la teoría fundamentada. Colombia: Universidad de Antioquia.

Vernon, S. (2004). Tres distintos enfoques en las propuestas de alfabetización inicial. En A. Pellicer y S. Vernon (comps.), Aprender y enseñar la lengua escrita en el aula. México: SM.

Viramontes, E., Morales, L. A., y Burrola, L. M. (2011). Aprendizaje de la escritura en lengua no materna: casos de niños tarahumaras. Revista Electrónica Educare, 15(1), 223-241. Recuperado de: https://www.redalyc.org/articulo.oa?id=194118804018.

Vygotsky, L. S. (1979). El desarrollo de los procesos psicológicos superiores. Buenos Aires: Grijalbo.

Cómo citar este artículo:

Viramontes Anaya, E., y Porras Palacio, A. (2020). Alfabetización inicial bilingüe e inclusión en la escuela primaria regular. RECIE. Revista Electrónica Científica de Investigación Educativa, 5(1), pp. 319-328. doi: doi. org/10.33010/recie.v5i1.948. 